\title{
How to Cut into a Material More Smoothly
}

\author{
A theory confirmed by experiments explains what has been an \\ unpredictable cutting process.
}

By Mark Buchanan

S licing through a solid can turn out a continuous band of liberated material or a disorderly sequence of fractured shards. Now researchers have shown that there is a universal transition between these two different ways materials come apart when cut [1]. Others have noted that the depth of the cutting tool determines the material's response, but the new theory explains why the transition occurs and predicts the depth at which the transition takes place for any relevant material. The theory is supported by new experiments with several different plastics as well as previous data from experiments on other materials. This new understanding may

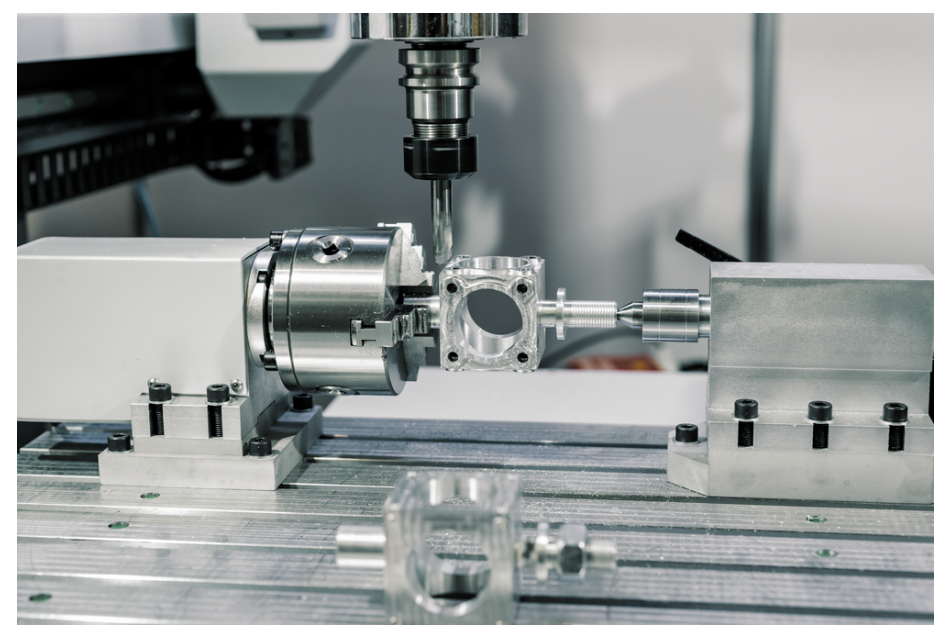

By a thousand cuts. A computer-controlled machining tool makes a metal part by cutting away bits of material. A new theory backed up by experiments explains why the depth of the cutting tool determines whether the excess material comes out as a continuous band or as a random sequence of chunks. (See videos below.) Credit: nordroden/stock.adobe.com

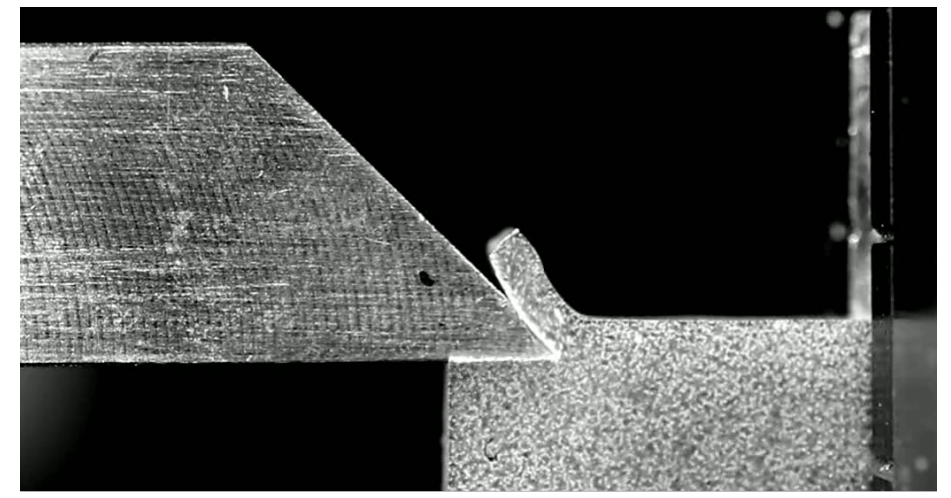

Cutting of polyoxymethylene (POM) at this tool angle and depth produces a continuous strip. (Video is sped up by about four times.) Credit: R. Aghababaei et al. [1]

allow manufacturers to design tools that achieve a better surface finish.

Lack of control is a serious problem for any precision cutting process. Cutting sometimes yields up a continuous band of smoothly sliced material, but the process is often more irregular, prone to producing many chips that break off, says Ramin Aghababaei, a specialist in mechanical and production engineering at the University of Aarhus in Denmark. Chipping can break the cutting tool and can reduce the smoothness of cuts, so researchers would like to know how to avoid it, he says. "How to control the size and shape of chips has been a longstanding mystery that often gets in the way of efficiently producing a high quality finish on cut materials." 


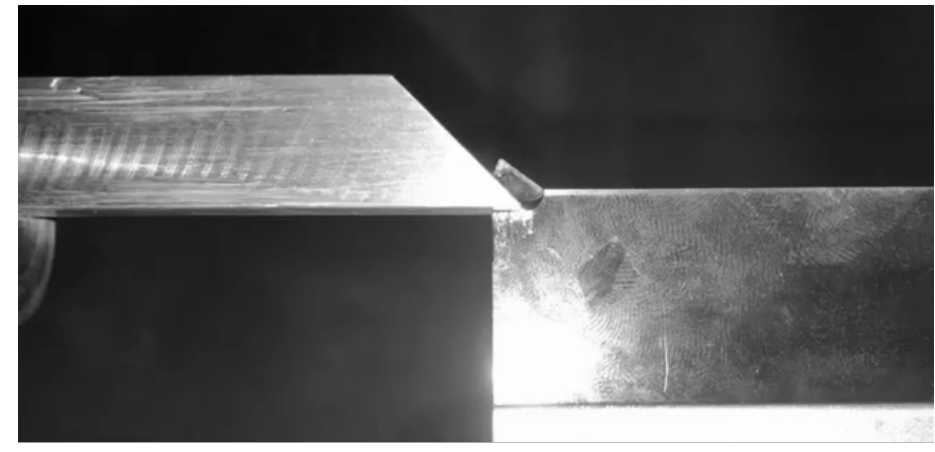

Cutting of polymethyl methacrylate (PMMA) with this tool angle and depth produces segmented chips that break off. (Video is sped up by about 2.5 times.)

Credit: R. Aghababaei et al. [1]

For many years, researchers have observed an abrupt transition between the two distinct behaviors with increasing depth of a cutting tool, but the precise conditions and material properties that determine the transition point were not known. To find out, Aghababaei and colleagues theoretically analyzed the two modes of cutting, deriving equations for the key forces produced inside a material by a cutting tool. The forces depend on the depth of the cut, on the physical properties of the material, and on the cutting geometry, especially the angle of the front edge of the cutting tool.

Based on this analysis, Aghababaei and his colleagues predicted the specific cutting depth at which the transition from one cutting mode to the other should occur. With shallower cuts, the material responds through deformation (stretching and squeezing), with the removed material remaining unbroken because the forces never become large enough to initiate fracture. However, beyond the critical depth, the forces become too large, and the material fractures, with chips breaking off.

The researchers then tested-and verified-this simple picture in experiments. With a mechanical controller and a sharp tool, they made a series of cuts into blocks, each made from one of three different plastic materials-one highly ductile, another brittle, and a third with intermediate properties. For the two more brittle materials, the cutting process turned out smooth strips when the cutting depth was sufficiently small, then produced multiple chips once the depth reached the critical value. For the most ductile plastic, the team was limited by their equipment and couldn't cut deep enough to observe the multichip behavior.

The team's theory provided a good prediction of the critical depth for each of the materials, with the critical-depth value becoming smaller with increasing brittleness. The researchers also compared their predictions with a number of previous studies of a wide range of materials. "For most cases," says Aghababaei, "our proposed model does remarkably well in predicting the way cutting occurs."

The model predicts that the critical cutting depth also depends on the temperature of the material, as most materials are more ductile when warmer, Aghababaei says. The team demonstrated this relationship in a further experiment that compared room temperature butter with frozen butter. At the same cutting depth, they observed chipping behavior for the cold sample and smooth cutting for the warmer sample. The results suggest that temperature is another variable that engineers could use to exert precise control over the nature of cutting in a wide range of materials.

"This paper is quite exciting," says engineering researcher Derek Warner of Cornell University. "It's a nice example of applying basic scientific understanding to arrive at a robust yet simple model with real-world value. Accomplishing such feats is difficult, and hence rare."

Mark Buchanan is a freelance science writer who splits his time between Abergavenny, UK, and Notre Dame de Courson, France.

\section{REFERENCES}

1. R. Aghababaei et al., "Cutting depth dictates the transition from continuous to segmented chip formation," Phys. Rev. Lett. 127, 235502 (2021). 